Quim. Nova, Vol. 33, No. 10, 2266-2271, 2010

\title{
CONSTRUÇÃO DE ESPECTRÔMETRO DE EMISSÃO ATÔMICA COM ATOMIZAÇÃO ELETROTÉRMICA EM FILAMENTO DE TUNGSTÊNIO (WCAES) ${ }^{\#}$
}

\author{
Mário H. Gonzalez, Luana N. Santos e Joaquim A. Nóbrega* \\ Departamento de Química, Universidade Federal de São Carlos, CP 676, 13560-970 São Carlos - SP, Brasil \\ Fábio R. P. Rocha \\ Centro de Energia Nuclear na Agricultura, Universidade de São Paulo, 13416-000 Piracicaba - SP, Brasil
}

Recebido em 28/2/10; aceito em 27/9/10; publicado na web em 3/11/10

\begin{abstract}
CONSTRUCTION OF A TUNGSTEN COIL ATOMIC EMISSION SPECTROMETER (WCAES). It is here discussed the development of a low cost analytical instrument with capacity for metals determination using atomic emission measurements in an electrothermal atomization system with a tungsten coil atomizer. The main goal was to show a new frontier for using this atomizer and to demonstrate that the simple instrumental arrangement here proposed has potential for portability and for solving analytical tasks related to metals determination. Atomic emission of calcium was selected for the adjustment of instrumental parameters and to evaluate the main characteristics of the lab-built instrument. Cobalt was determined in medicines and one alloy to demonstrate its feasibility.
\end{abstract}

Keywords: atomic emission spectrometry; tungsten coil atomic emission spectrometry (WCAES); instrumentation.

\section{INTRODUÇÃO}

Medidas de emissão são realizadas para vários propósitos. $\mathrm{O}$ comprimento de onda das linhas de emissão espectral traz informações sobre a estrutura de átomos e moléculas. As intensidades relativas das linhas e bandas espectrais também são usadas para análises quantitativas. A espectrometria de emissão atômica em chama (FAES) foi introduzida por Kirchhoff e Bunsen no final do século XIX para análise qualitativa de elementos metálicos. Lundegardh introduziu um detector fotoelétrico e um dispositivo de leitura direta para a determinação de potássio em amostras agronômicas no final da década de 20 do século XX. ${ }^{1}$

Vários dispositivos são empregados como fonte de excitação, sendo o plasma de argônio a alternativa mais difundida. As altas temperaturas e estabilidade alcançadas pelo plasma ampliam a capacidade analítica das chamas químicas convencionais, possibilitando determinações multielementares de cerca de 70 espécies atômicas. A principal desvantagem dessa fonte de excitação é o elevado custo para aquisição e manutenção. ${ }^{2}$

Uma alternativa é o emprego de superfícies metálicas como atomizadores em substituição ao tubo de grafite e plasma. Esses atomizadores apresentam características favoráveis, tais como não formação de carbetos, elevada vida útil, rápido aquecimento através de uma fonte elétrica de baixa potência, menor emissão de radiação nas regiões do vísivel e ultravioleta do espectro eletromagnético, ${ }^{3}$ baixo custo de aquisição, baixo consumo de energia, possibilidade de trabalhar com temperaturas de até 3200 C, ausência de efeitos de memória, devido à não porosidade da superfície e, não necessidade de sistema de refrigeração., ${ }^{3,4}$

Vários elementos foram empregados como atomizadores metálicos, sendo os principais: molibdênio, platina, tântalo e tungstênio. ${ }^{4}$ O tungstênio apresenta o maior ponto de fusão (3680 K) e a menor pressão de vapor entre todos os metais. ${ }^{5,6}$ Além disso, apresenta alta resistência química, sendo atacado significativamente apenas pela mistura de ácidos fluorídrico e nítrico ou por fusão alcalina oxidante. ${ }^{7}$ Esses dispositivos requerem adição de $\mathrm{H}_{2}$ e argônio como gás de purga visando proteger o metal de processos de oxidação e, consequentemente, ampliar a vida útil do atomizador, a precisão e a sensibilidade.

\footnotetext{
*e-mail: djan@terra.com.br

\#Artigo em homnagem ao Prof. Hans Viertler
}

Na década de 70 iniciaram-se os esforços para o desenvolvimento de um espectrômetro de absorção atômica com um filamento de tungstênio como atomizador eletrotérmico. ${ }^{8-10}$ A baixa potência desses filamentos $(24 \mathrm{~W})$ e as limitações eletrônicas para a aquisição de rápidos sinais transientes dificultaram maiores avanços. Esses estudos somente foram retomados a partir do trabalho de Berndt e Schaldach, em 1988, usando um filamento de tungstênio de $150 \mathrm{~W}$ adaptado em um espectrômetro de absorção atômica com capacidade para medida de sinais transientes originados por nuvens atômicas geradas por atomização eletrotérmica. ${ }^{4}$ Os principais atrativos demonstrados para esse atomizador foram o baixo custo e a elevada sensibilidade. Conforme pode ser avaliado pela literatura, ${ }^{11-14}$ os estudos passaram a ser principalmente desenvolvidos no Brasil, Chile e Estados Unidos.

As principais limitações observadas para medidas de absorção/emissão atômica com atomização eletrotérmica em filamento de tungstênio são a falta de um equipamento comercial e a ocorrência de interferências. ${ }^{15,16}$ Por outro lado, o entendimento e a resolução de interferências ainda requerem estudos de cunho fundamental, tal como o de Queiroz et al., ${ }^{17}$ sobre medidas de temperaturas da fase gasosa e da superfície metálica para um atomizador eletrotérmico com filamento de tungstênio.

A maioria dos estudos com atomizadores metálicos envolveu medidas de absorção atômica, sendo que poucos estudos realizados no Japão investigaram o uso de tubos de molibdênio, ${ }^{18,19}$ ou de tungstênio, ${ }^{20,21}$ para medidas de emissão atômica. Esse limitado número de estudos pode ser justificado pela saturação do detector, no passado geralmente constituído por uma válvula fotomultiplicadora, devido à intensa emissão de radiação pelo tubo metálico mantido sob temperaturas de até $3000{ }^{\circ} \mathrm{C}$ na etapa de atomização. Por outro lado, o atrativo das medidas de emissão reside na possibilidade de construção de um equipamento ainda mais simples que o empregado em medidas de absorção, sem a necessidade de uma fonte de radiação e respectiva fonte de alimentação. Além disso, medidas de emissão atômica possibilitam determinações multielementares.

Em 2005, sinais de emissão atômica foram observados em um sistema de absorção atômica com filamento de tungstênio usando um arranjo de dispositivos de carga acoplada (CCD) e um espectrômetro de alta resolução Czerny-Turner. Estudando-se mais detalhadamente esses sinais propôs-se uma nova técnica denominada espectrometria de emissão atômica com filamento de tungstênio (WCAES). ${ }^{22}$ 
Algumas aplicações com WCAES foram então descritas. Rust et $a .^{23}$ avaliaram as figuras de mérito para a determinação de 11 elementos, i.e., Al, Co, Cr, Dy, Ga, K, Mn, Pb, Rb, Sc e Yb. Donati et al. ${ }^{24}$ propuseram a determinação simultânea de 14 lantanídeos, a determinação simultânea de $\mathrm{Cr}, \mathrm{Ga}$, In e $\mathrm{V}, 25$ e a aplicação pioneira de um atomizador metálico com filamento de tungstênio para determinação de iodo. ${ }^{26}$ Um novo arranjo empregando dois filamentos de tungstênio foi proposto para a técnica WCAES. ${ }^{27}$ Duas fontes de corrente elétrica constante e um dispositivo de carga acoplada (CCD) foram empregados para determinação de elementos refratários em amostras de água (Ba, Sr, Ti e V).

A indisponibilidade comercial de equipamentos para medidas por WCAES pode dificultar o desenvolvimento de novas aplicações e o envolvimento de novos grupos de pesquisa. Dessa forma, o estudo aqui descrito aborda o desenvolvimento de um espectrômetro de emissão atômica com atomizador eletrotérmico com filamento de tungstênio aplicável para a determinação de metais. O arranjo instrumental teve como base uma rede de difração associada a um sistema de detecção constituído por um arranjo linear de dispositivos de carga acoplado. $\mathrm{O}$ filamento de tungstênio foi aquecido por uma fonte de tensão constante com baixa potência $(150 \mathrm{~W})$. Medidas de emissão atômica de cálcio foram adotadas como modelo para ajustes instrumentais e para avaliar as características da WCAES. A viabilidade da determinação de cobalto em medicamentos e liga metálica também foi avaliada.

\section{PARTE EXPERIMENTAL}

\section{Instrumentação}

O equipamento para medidas por WCAES foi construído com os seguintes componentes:

- Rede de difração com 3648 linhas/mm associada a um arranjo de detectores de estado sólido (Ocean Optics, HR 4000, Dunedin, FL, EUA): dimensões de 149 (comprimento) x 105 (largura) x $45 \mathrm{~mm}$ (altura), com faixa de detecção entre $330-425 \mathrm{~nm}, 3648$ pixels, fenda de entrada de $50 \mu \mathrm{m}$ e resolução espectral da ordem de $0,02 \mathrm{~nm}$;

- Fonte elétrica com tensão controlada, produzida pela Anacom Científica (São Bernardo do Campo, SP). Sua principal característica é o emprego de um sistema de retroalimentação para manter constante a tensão aplicada ao filamento independentemente da variação da temperatura e, consequentemente, da resistência do metal ao longo do ciclo de aquecimento. Esse processo é mais crítico na etapa de secagem, sendo que no início a quantidade de calor resultante é consumida para remover o solvente e, em seguida, ocorre um aumento da temperatura da superfície do filamento;

- Computador para aquisição e tratamento de dados;

- Filamentos de tungstênio (Osram, 150 W, Dortmund, Alemanha); - Mesa, tripés, trilho óptico e lente focalizadora para montagem do instrumento (Melles-Griot, Albuquerque, NM, EUA);

- Forno de vidro borossilicato com janelas de quartzo, eletrodos de cobre e suporte de politetrafluoretileno para adaptação do filamento de tungstênio e espectrômetro. Esses dispositivos foram construídos nas oficinas de hialotecnia e mecânica do Departamento de Química da UFSCar;

- Rotâmetro graduado entre 0,3-3,5 L min-1 empregado para o controle da vazão da mistura gasosa.

\section{Soluções e mistura gasosa}

As soluções de referência foram preparadas a partir da diluição de soluções estoque monoelementares contendo $1000 \mathrm{mg} \mathrm{L}^{-1} \mathrm{Ca}$ e Co (Aldrich, Milwaukee, WI, EUA). As soluções foram preparadas em meio de $\mathrm{HNO}_{3} 0,14$ mol L-1. Utilizou-se para proteção do filamento de tungstênio e formação da nuvem atômica de cálcio uma mistura gasosa composta por argônio e hidrogênio $90: 10 \mathrm{v} \mathrm{v}^{-1}$ (White Martins, Sertãozinho, SP, Brasil).

\section{RESULTADOS E DISCUSSÃO}

\section{Montagem e desenvolvimento do protótipo}

O forno de vidro borossilicato com janelas de quartzo foi construído conforme representado na Figura 1.

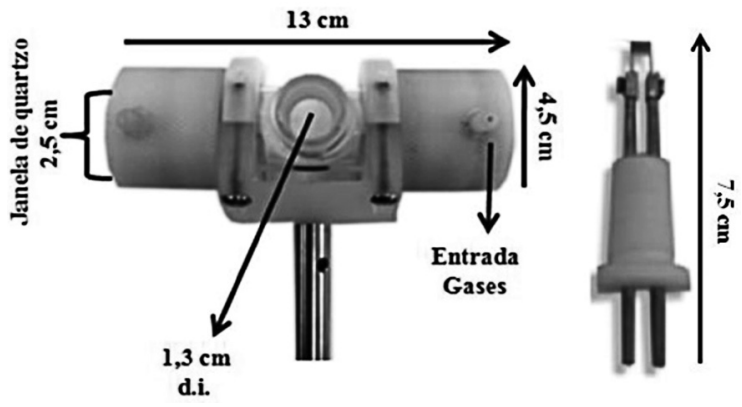

Figura 1. Forno de borossilicato para filamento de tungstênio

Após o desenvolvimento do forno de borossilicato, do eletrodo para suporte do atomizador metálico, do suporte para o espectrômetro e dos ajustes necessários na fonte de potência, o protótipo foi construído de acordo com a Figura 2.

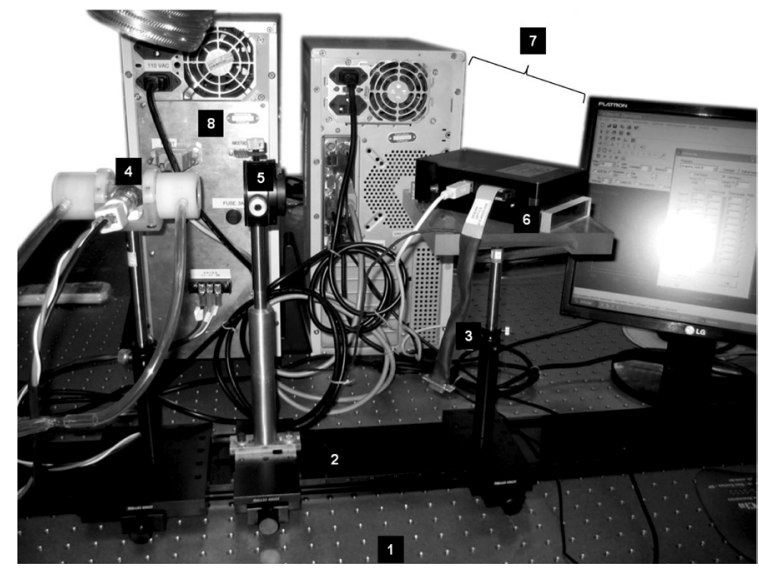

Figura 2. Espectrômetro de emissão atômica com atomizador de filamento de tungstênio. 1) mesa óptica; 2) trem óptico; 3) trilhos ópticos; 4) forno de borossilicato; 5) lente colimadora; 6) espectrômetro e detector CCD; 7) computador e 8) fonte de potência controlada

A introdução da solução da amostra sobre a superfície do tungstênio é possível devido à configuração helicoidal do filamento e à tensão superficial do solvente. A introdução da amostra é feita com adequada precisão e distribuição homogênea da solução no atomizador usando uma micropipeta manual ou um amostrador automático. Os volumes introduzidos variam de 10 a $25 \mu \mathrm{L}$, podendo ser introduzidas soluções de digeridos ácidos, suspensões e amostras líquidas diretamente na superfície do filamento.

O programa de aquecimento (Tabela 1), que compreende as etapas de secagem, pirólise e atomização, é crítico no desenvolvimento de qualquer procedimento analítico baseado em atomização eletrotérmica. Eventuais problemas nessa etapa acarretam perda de analitos por volatilização, diminuindo a sensibilidade e repetibilidade. 
Oliveira et al. ${ }^{28}$ avaliaram programas de aquecimento para determinação de elementos voláteis utilizando espectrometria de absorção atômica com atomização eletrotérmica em filamento de tungstênio e observaram que o uso de uma rampa invertida de aquecimento evitava um aquecimento excessivo do filamento imediatamente após a evaporação do solvente, devido às mudanças da resistência do filamento de tungstênio.

Tabela 1. Programa de aquecimento para medidas por WCAES

\begin{tabular}{ccc}
\hline Etapa & Tensão aplicada (V) & Tempo (s) \\
\hline 1 & 0,70 & 10 \\
2 & 0,65 & 5 \\
3 & 0,60 & 5 \\
4 & 0,50 & 5 \\
5 & 0,40 & 5 \\
6 & 0,30 & 5 \\
7 & 13,0 & 3 \\
8 & 0 & 20 \\
\hline
\end{tabular}

Newton e Davis ${ }^{29}$ mostraram que a adição de $20 \% \mathrm{v} \mathrm{v} \mathrm{v}^{-1}$ de hidrogênio ao gás de purga (nitrogênio ou argônio) aumentou a vida útil do filamento, reduziu processos de absorção não atômica e diminuiu interferências químicas. Também observaram que a magnitude das interferências dependia do programa de aquecimento. Suzuki e $\mathrm{Ohta}^{30}$ descreveram essas mesmas vantagens, ressaltando a proteção do atomizador pela oxidação de traços de oxigênio na câmara de atomização.

A radiação emitida pela excitação térmica da nuvem atômica na etapa de atomização é focalizada para a fenda de entrada do monocromador com o auxílio de uma lente colimadora. A radiação atinge a superfície do elemento dispersor (rede de difração), sendo direcionada para o sistema de detecção de estado sólido (CCD). Foram realizados experimentos relacionados à distância e altura de observação entre o atomizador e o espectrômetro para se estabelecer a melhor região de observação (i.e., maiores intensidades das linhas de emissão) para cálcio.

\section{Potencialidade da WCAES na determinação de cálcio em amostras de águas}

A primeira etapa realizada foi a busca das linhas de emissão para o cálcio e suas intensidades relativas. Visando a quantificação de cálcio, as linhas de emissão em 393,3 e 422,7 nm foram selecionadas com base na consulta das intensidades relativas no banco de dados espectrais do National Institute of Standards and Technology. ${ }^{31}$ Após a escolha das linhas de emissão, a distância entre atomizador, lentes e espectrômetro foi avaliada, de modo a minimizar a radiação de fundo emitida pelo filamento (BG) que alcança o sistema de detecção e posicionar corretamente a imagem do filamento na entrada da fenda espectral do espectrômetro. A distância ajustada entre o atomizador e a lente colimadora e dessa para o espectrômetro foi de $14,5 \mathrm{~cm}$, que corresponde ao ponto focal da lente. Observandose o BG para uma tensão aplicada de $10 \mathrm{~V}$, constatou-se que nesse posicionamento a radiação emitida estava focalizada na fenda de entrada do espectrômetro.

A intensidade de emissão é altamente dependente da posição do atomizador em relação ao espectrômetro. Maiores intensidades são observadas quando o filamento de tungstênio é alinhado no centro da entrada do monocromador sendo, entretanto, a emissão devida principalmente ao sinal de fundo. Na presença do analito, a separação temporal entre o sinal de emissão (IS) e o BG é crítica. Devido à alta taxa de aquecimento do filamento de tungstênio, a emissão do analito antecede a intensidade máxima do sinal de fundo. A magnitude do sinal de fundo aumenta com o tempo de atomização, o que dificulta medidas de emissão atômica devido à saturação do detector. Esse aspecto é mais critico para elementos refratários, que requerem maior temperatura para atomização e excitação.

O ajuste da região de observação é crítico e deve-se considerar a diferença de temperatura entre a superfície do filamento de tungstênio e a fase gasosa. Estudos evidenciaram gradientes de temperaturas de até $1000^{\circ} \mathrm{C}$ entre a fase gasosa e a superfície do filamento, dependendo da tensão aplicada e da altura de observação. ${ }^{17} \mathrm{~A}$ altura de observação ideal é aquela na qual o sinal de fundo proveniente do filamento de tungstênio é minimizado, enquanto a emissão do analito na fase gasosa é direcionada para a entrada da fenda do espectrômetro.

É possível posicionar o filamento horizontalmente (posição axial) ou verticalmente (posição radial) à entrada do espectrômetro (Figura 3). Para o elemento cálcio foi inicialmente utilizado o filamento na posição radial, porém não foi possível detectar os sinais de emissão. Utilizou-se então o filamento na posição horizontal e variou-se gradualmente a posição relativa em relação à entrada do espectrômetro, desde o centro até atingir a extremidade externa. Na posição mostrada na Figura 3a, observou-se a formação de picos de emissão nas regiões de 422,7 e 393,3 nm e obteve-se separação temporal adequada entre o sinal de emissão do Ca e o BG.

(a) (b)

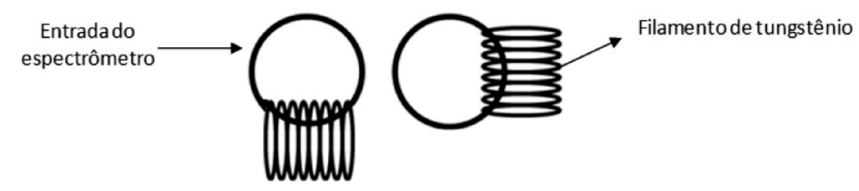

Figura 3. Posicionamento do filamento de $W$ em relação à fenda de entrada do espectrômetro (a) visão axial (b) visão radial

O efeito da tensão aplicada na etapa de atomização foi avaliado realizando-se medidas entre 10 e $14 \mathrm{~V}$ para solução $25 \mathrm{mg} \mathrm{L}^{-1} \mathrm{Ca}$ (Figura 4). As tensões de 11 e $12 \mathrm{~V}$ geraram sinais de emissão pouco intensos. A partir de $13 \mathrm{~V}$, foi verificado o aumento das intensidades relativas do sinal de emissão nos comprimentos de onda selecionados. Aplicando-se uma tensão de $15 \mathrm{~V}$, as intensidades foram similares aos sinais de emissão de fundo do filamento de tungstênio e, além disso, considerando-se o maior desgaste do filamento, essa condição não foi adotada. Assim, optou-se pela aplicação de uma tensão de $14 \mathrm{~V}$ (Figura 4).

A sensibilidade do sistema de detecção foi avaliada empregando-se diferentes tempos de integração. Nesse estudo a separação temporal dos sinais de emissão do analito (IS) e BG foi criticamente avaliada, visando a diferenciação entre ambos. A tensão aplicada foi de $14 \mathrm{~V}$ e a vazão do gás de purga foi de $1,0 \mathrm{~L} \mathrm{~min}^{-1}$. A Figura 5 mostra o perfil de atomização para solução de cálcio $25 \mathrm{mg} \mathrm{L}^{-1}$ para tempos de integração $100,200,300$ e $500 \mathrm{~ms}$. Verificou-se que para $100 \mathrm{~ms}$ de integração a intensidade do sinal de emissão para Ca em $422,7 \mathrm{~nm}$ foi inferior aos demais tempos de integração. A intensidade do sinal de emissão foi duplicada para tempo de integração de $200 \mathrm{~ms}$ e diminuiu para tempos superiores de integração. Esse parâmetro para coleta dos dados reflete um compromisso cuidadoso com a aquisição de um sinal transiente de uma nuvem contendo átomos no estado fundamental e excitado, cujo tempo de duração é geralmente inferior a $1 \mathrm{~s}$ dependendo da volatilidade do analito. O perfil observado para o comprimento de onda de 393,3 nm foi análogo ao obtido para $422,7 \mathrm{~nm}$.

O efeito da vazão do gás de purga, mistura contendo $10 \%$ de $\mathrm{H}_{2}$ e $90 \% \mathrm{v} \mathrm{v}^{1}$ de Ar, foi avaliado. A presença do gás é necessária para 


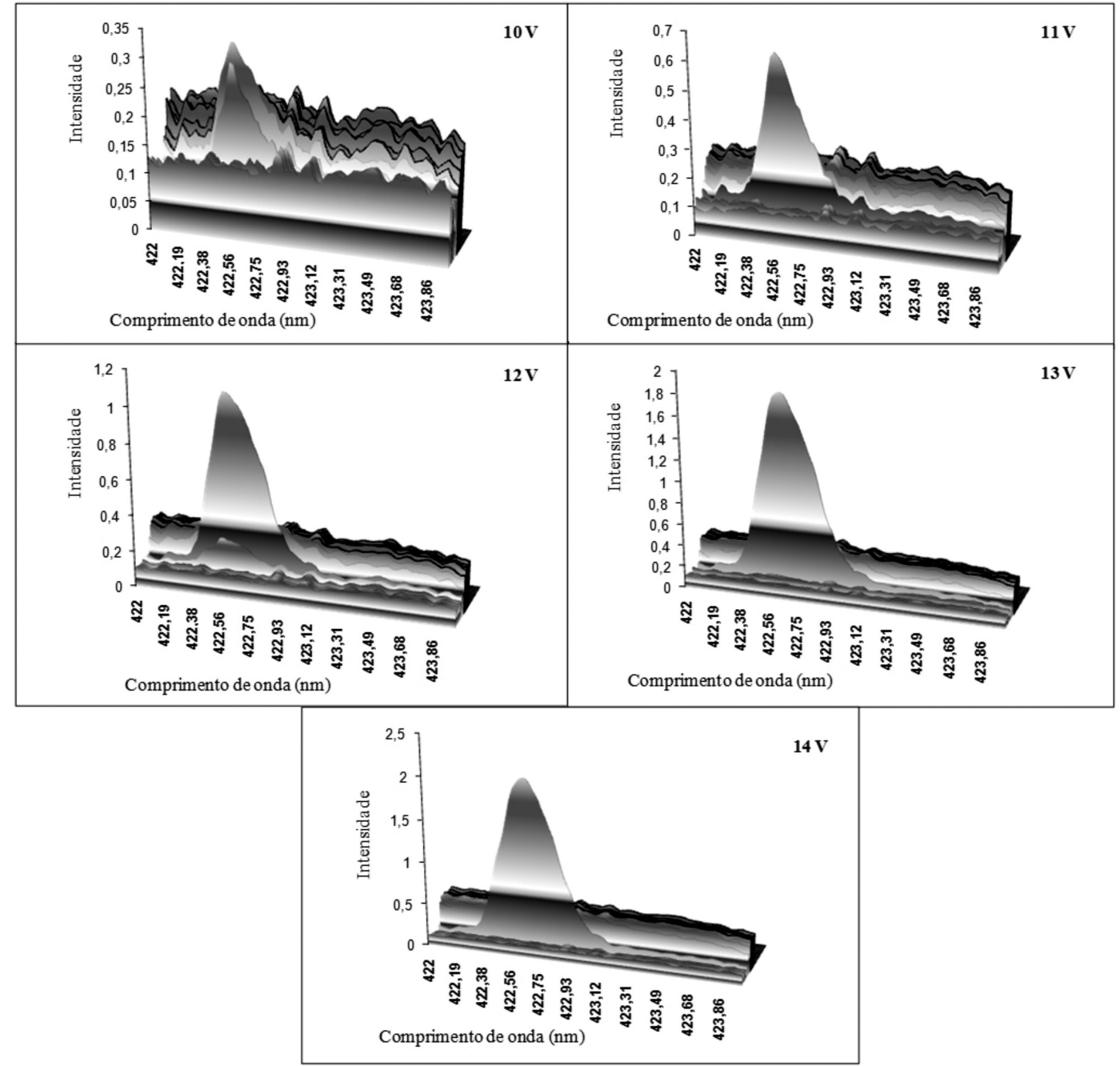

Figura 4. Perfis de atomização para Ca $100 \mathrm{mg} \mathrm{L}^{-1}$ para diferentes tensões aplicadas. Máximo de emissão em $422,7 \mathrm{~nm}$

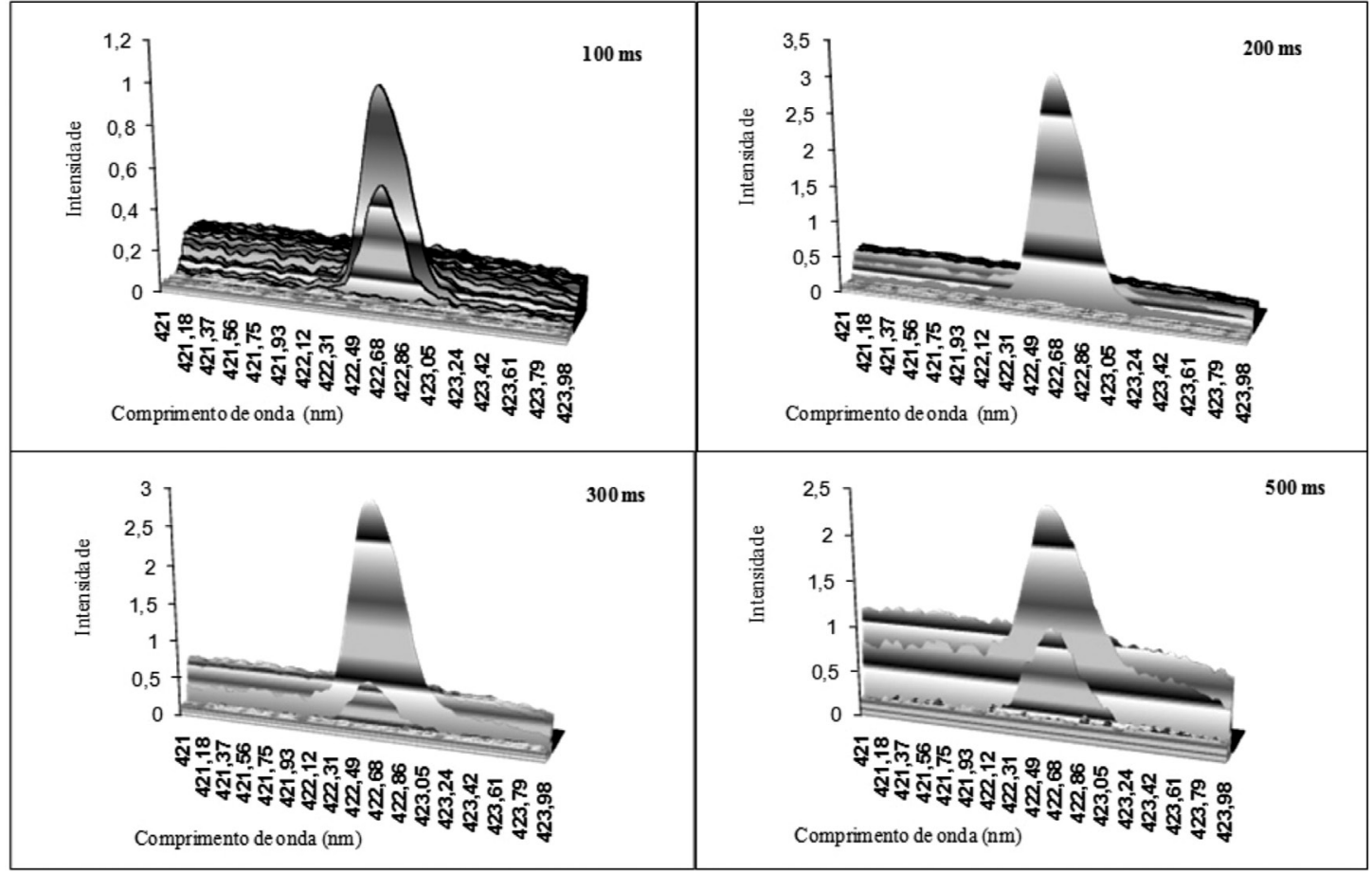

Figura 5. Perfis de atomização para Ca $25 \mathrm{mg}$ L $^{-1}$ para diferentes tempos de integração, com tensão aplicada de $14 \mathrm{~V}$. Máximo de emissão em $422,7 \mathrm{~nm}$ 


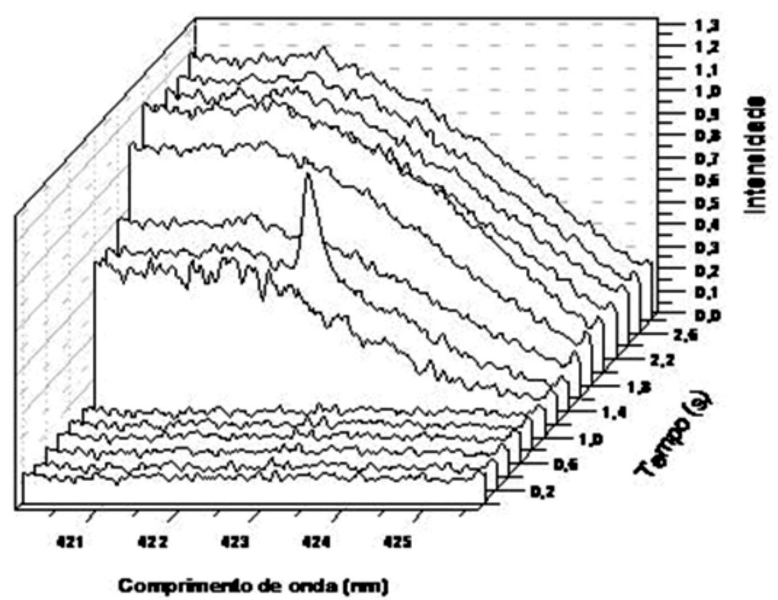

(a)

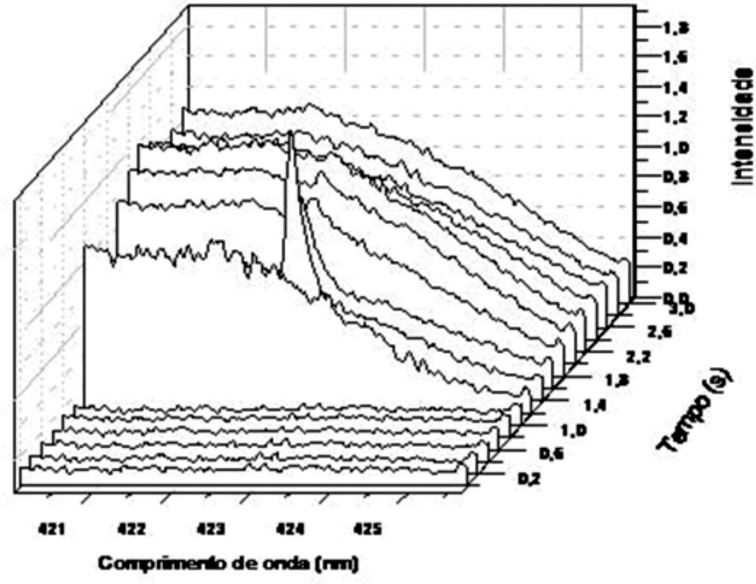

(b)

Figura 6. Sinais de emissão atômica para Ca (a) 1,0 $\mathrm{mg} \mathrm{L}^{-1}$ e (b) 2,0 $\mathrm{mg} \mathrm{L}^{-1}$ no comprimento de onda de $422,7 \mathrm{~nm}$

a formação de uma atmosfera redutora protegendo o filamento de oxidação, evitando assim o desgaste excessivo e promovendo a atomização do analito. A vazão do gás utilizada foi de $1,0 \mathrm{~L} \mathrm{~min}^{-1}$, uma vez que para vazões maiores ocorreu a diminuição nas intensidades de emissão pela diluição da nuvem atômica. Vazões menores causaram diminuição da vida útil do filamento de tungstênio. A Figura 6 apresenta a formação da nuvem atômica para duas concentrações (a) 1,0 mg L $\mathrm{me}^{-1}$ e (b) 2,0 mg L-1 usando as condições experimentais estabelecidas.

A aquisição de dados foi iniciada $1 \mathrm{~s}$ antes do início da etapa de atomização para evitar possíveis problemas de sincronismo entre os programas de controle da fonte de potência e do espectrômetro. O aparecimento do sinal de emissão atômica para o cálcio ocorreu entre 400 e 600 ms. Em 700 ms, observou-se a saturação do sistema de detecção causado pelo BG. Foram coletados espectros a cada $100 \mathrm{~ms}$ durante $2 \mathrm{~s}$, gerando 20 espectros para cada medida realizada para $\mathrm{Ca}$.

Uma dificuldade observada na WCAES é que o alinhamento da nuvem atômica com a fenda de entrada do espectrômetro é crítico e específico para cada analito. Assim, a melhor posição obtida para medidas de Ca pode não ser a melhor posição para o restante dos concomitantes. Apesar de esse aspecto dificultar determinações quantitativas simultâneas de vários analitos, é possível aproveitá-lo para a minimização de interferências espectrais.

\section{Aplicação analítica: determinação de Co em medicamentos e liga metálica}

Inicialmente buscou-se avaliar os sinais observados nas linhas mais intensas de emissão para o cobalto $(340,5$; 344,5; 345,4 e 350,3 $\mathrm{nm})$. A separação temporal entre o sinal de emissão do cobalto e o BG foi otimizada de acordo com a Figura 3a. Após o estabelecimento da melhor altura de observação, os efeitos causados pela vazão do gás de atomização/proteção $\left(0,7-1,3 \mathrm{~L} \mathrm{~min}^{-1}\right)$ e pela tensão aplicada (8-14 V) foram avaliados utilizando-se metodologia de superfície de resposta através de uma matriz Doehlert. As superfícies de resposta obtidas para os experimentos referentes aos quatro comprimentos de onda do cobalto evidenciaram que as melhores condições foram obtidas quando foram utilizadas maiores intensidades na corrente (i.e., maior temperatura) e menores vazões do gás de purga. Observando os gráficos de resíduos (preditos $\mathrm{X}$ observados) pode-se notar que o modelo se ajusta bem aos dados. Isso pode ser confirmado também através da análise de variância, sendo que todos os modelos gerados (um modelo para cada comprimento de onda) apresentaram ajuste adequado. Assim, o desenho Doehlert foi eficiente para estabelecer as condições ótimas de trabalho. Os valores otimizados foram: tensão aplicada de $14 \mathrm{~V}$ e vazão de gás de purga de $0,9 \mathrm{~L} \mathrm{~min}^{-1}$.

A faixa linear de calibração foi de 0,5 a $50 \mathrm{mg} \mathrm{L}^{-1}$. O procedimento proposto tem correlação linear em todos os comprimentos de onda, variando o coeficiente de correlação (R) entre 0,9997-0,9999. Os limites de detecção (LOD) para as 4 linhas $(340,5 ; 344,5 ; 345,4$ e $350,3 \mathrm{~nm}$ ) foram 0,$36 ; 0,34 ; 0,33$ e $0,48 \mathrm{mg} \mathrm{L}^{-1}$, respectivamente, e de quantificação (LOQ) de 1,2; 1,1; 1,1 e 1,6 $\mathrm{mg} \mathrm{L}^{-1}$, respectivamente.

As amostras de medicamentos e liga metálica foram decompostas empregando-se radiação micro-ondas em meio de ácido nítrico e ácidos nítrico e fluorídrico, respectivamente. Os digeridos foram diluídos conforme a faixa linear de calibração, sendo que essa diluição também minimizou o ataque do ácido fluorídrico residual ao filamento de tungstênio. A vida útil típica do filamento de tungstênio foi de 200 ciclos de aquecimento. Os teores de cobalto nos digeridos diluídos foram determinados por WCAES e por ICP OES como método comparativo. Os resultados estão apresentados na Tabela 2. Não ocorreram diferenças significativas para um nível de confiança de $95 \%$, demonstrando assim a exatidão da WCAES.

Tabela 2. Teores médios e desvios padrão $(n=3)$ de cobalto determinados por WCAES e ICP OES em digeridos de medicamentos e liga metálica

\begin{tabular}{|c|c|c|c|c|c|}
\hline & \multicolumn{4}{|c|}{ WCAES } & \multirow[t]{2}{*}{ ICP OES } \\
\hline & $340,5 \mathrm{~nm}$ & $344,5 \mathrm{~nm}$ & $345,4 \mathrm{~nm}$ & $350,3 \mathrm{~nm}$ & \\
\hline & \multicolumn{5}{|c|}{$* \mathrm{mg} \mathrm{L}^{-1} / * * \mathrm{mg} \mathrm{kg}^{-1}$} \\
\hline Medicamento A* & $5,4 \pm 0,4$ & $5,3 \pm 0,6$ & $5,6 \pm 0,5$ & $5,3 \pm 0,5$ & $5,8 \pm 0,4$ \\
\hline Medicamento $\mathrm{B}^{* *}$ & $11,6 \pm 1,3$ & $11,9 \pm 1,2$ & $11,3 \pm 0,8$ & $11,2 \pm 1,1$ & $11,5 \pm 0,8$ \\
\hline Liga metálica** & $15,2 \pm 1,1$ & $15,3 \pm 1,0$ & $15,2 \pm 1,0$ & $15,1 \pm 1,3$ & $15,3 \pm 0,9$ \\
\hline
\end{tabular}

\section{CONCLUSÃO}

Este trabalho apresenta o desenvolvimento de um instrumento analítico de baixo custo com capacidade para determinação de metais empregando medidas de emissão atômica. Busca-se abrir uma nova fronteira para uso desse atomizador e demonstrar que o arranjo tem potencial para o desenvolvimento de um equipamento portátil que poderá complementar o trabalho analítico atualmente efetuado com técnicas tradicionais.

A WCAES apresenta algumas vantagens quando comparada com as técnicas tradicionais, tais como portabilidade, baixo custo, 
capacidade de análise com pequenos volumes de amostra e possibilidade de análises multielementares, que a tornam uma alternativa para aplicações analíticas em medidas in situ.

\section{AGRADECIMENTOS}

À Coordenação de Aperfeiçoamento de Pessoal de Nível Superior (CAPES) e ao Conselho Nacional de Desenvolvimento Científico e Tecnológico $(\mathrm{CNPq})$ pelas bolsas concedidas e à FAPESP pelo financiamento do Projeto $\mathrm{n}^{\circ}$. 2007/51448-0. Aos Srs. A. A. Sertori e E. de Almeida, funcionários do DQ/UFSCar, pela confecção de dispositivos ópticos e mecânicos. Ao Sr. K. Kamner (Osram, Alemanha) pelo fornecimento dos filamentos de tungstênio.

\section{REFERÊNCIAS}

1. Alkemade, C. T. J.; Hollander, T.; Snelleman, W.; Zeegers, P. J. T.; Metal Vapours in Flames, $1^{\text {st }}$ ed., Pergamon Press: Oxford, 1982.

2. Harris, D. C.; Quantitative Chemical Analysis, $4^{\text {th }}$ ed., W. H. Freeman, Pergamon Press: New York, 1995.

3. Berndt, H.; Schaldach, G.; J. Anal. At. Spectrom. 1988, 3, 709.

4. Giné, M. F.; Krug, F. J.; Sass, V. A.; Reis, B. F.; Nóbrega, J. A.; Berndt, H.; J. Anal. At. Spectrom. 1993, 8, 243.

5. Emsley, J.; The Elements, $1^{\text {st }}$ ed., Oxford: New York, 1989.

6. Weast, R. C.; Handbook of Chemistry and Physics, $64^{\text {th }}$ ed., Boca Raton: Florida, 1989.

7. Cotton, F. A.; Wilkinson, G.; Advanced Inorganic Chemistry, $5^{\text {th }}$ ed., Wiley: New York, 1988.

8. Piepmeier, E. H.; Williams, M.; Anal. Chem. 1972, 44, 1342.

9. Piepmeier, E. H.; Reid, R. D.; Anal. Chem. 1972, 48, 338.

10. Lund, W.; Larsen, B. V.; Anal. Chim. Acta 1974, 70, 299.

11. Nóbrega, J. A.; Silva, M. M.; Oliveira, P. V.; Krug, F. J.; Baccan, N.; Quim. Nova 1995, 18, 555.
12. Ribeiro, A. S.; Arruda, M. A. Z.; Cadore, S.; Quim. Nova 2002, 25, 396.

13. Hou, X.; Jones, B. T.; Microchem. J. 2000, 66, 115.

14. Hou, X.; Jones, B. T.; Spectrochim. Acta 2002, 57B, 659.

15. Oliveira, P. V.; Krug, F. J.; Silva, M. M.; Nóbrega, J. A.; Queiroz, Z. F.; Rocha, F. R. P.; J. Braz. Chem. Soc. 2000, 11, 136.

16. Queiroz, Z. F.; Krug, F. J.; Oliveira, P. V.; Silva, M. M.; Nóbrega, J. A.; Spectrochim. Acta, Part B 2002, 57, 49.

17. Queiroz, Z. F.; Oliveira, P. V.; Nóbrega, J. A.; Silva, C. S.; Rufini, I. A.; Sousa, S. S.; Krug, F. J.; Spectrochim. Acta, Part B 2002, 57, 1789.

18. Suzuki, M.; Ohta, K.; Talanta 1981, 28, 177.

19. Suzuki, M.; Ohta, K.; Yamakita, T.; Anal. Chem. 1981, 53, 1796.

20. Suzuki, M.; Ohta, K.; Anal. Chem. 1985, 57, 26.

21. Ohta, K.; Itoh, S.; Mizuno, T.; Talanta 1991, 38, 325.

22. Rust, J. A.; Nóbrega, J. A.; Calloway, C. P.; Jones, B. T.; Spectrochim. Acta, Part B 2005, 60, 589.

23. Rust, J. A.; Nóbrega, J. A.; Calloway Jr., C. P.; Jones, B. T.; Spectrochim. Acta, Part B 2006, 61, 225.

24. Donati, G. L.; Calloway, C. P.; Jones, B. T.; J. Anal. At. Spectrom. 2008, 23,361 .

25. Donati, G. L.; Kron, B. E.; Jones, B. T.; Spectrochim. Acta, Part B 2009 , 61,225 .

26. Donati, G. L.; Nóbrega, J. A.; Nascentes, C. C.; Jones, B. T.; Microchem. J. 2009, 93, 242.

27. Donati, G. L.; Calloway, C. P.; Jones, B. T.; J. Anal. At. Spectrom. 2009, 24, 1105.

28. Oliveira, P. V.; Nóbrega, J. A.; Catanho, M.; Luccas, P. O.; Quim. Nova 2000, 23, 706.

29. Newton, M. P.; Davis, D. G.; Anal. Chem. 2003, 47, 1975.

30. Suzuki, M.; Ohta, K.; Fresenius Z. Anal. Chem. 1982, 34, 313.

31. http://physics.nist.gov/PhysRefData/ASD/index.html, acessada em Março 2009. 